\title{
Un método automático de extracción de información de imágenes digitales basado en la detección de posicionamiento de marcas ópticas a partir de distancias euclidianas y redes neuronales
}

\author{
A method for the automatic extraction of information from digital \\ images based on the detection of the positioning of optical marks from \\ Euclidean distances and neural networks
}

Recibido: julio abril de 2016 | Revisado: mayo de 2016 | Aceptado: junio de 2016

\section{Christian DEL CARPIO ${ }^{1}$ Guillermo Kemper ${ }^{2}$}

1 Universidad de San Martín de Porres (USMP) Facultad de Ingeniería y Arquitectura- Escuela Profesional de Ingeniería Electrónica. Lima - Perú cdelcarpiod@usmp.pe

2 Universidad de San Martín de Porres (USMP) Facultad de Ingeniería y Arquitectura- Escuela Profesional de Ingeniería Electrónica. Lima - Perú gkemperv@usmp.pe

\begin{abstract}
A method of optical mark recognition using is presented in this investigation. Said method is oriented to the recognition of the code and annotation and of a student in an assessment booklet. The marks are generated in the booklet using a pen. Algorithms and image processing techniques such as spatial filtering, thresholding, segmentation, description and recognition through neural networks are used. The proposed method allows to automate the entry of academic grades into a database, without significantly altering the format of the booklets used in student evaluations. The results show that the proposed method achieves a maximum error of $2 \%$ in the recognition process.
\end{abstract}

Keywords: digital image processing, neural networks

\begin{abstract}
Resumen
Se plantea un método de reconocimiento de marcas ópticas utilizando una cámara WEB a través del presente trabajo. El mismo se orienta al reconocimiento del código y la nota de un estudiante en un cuadernillo de evaluación. Las marcas se generan en el cuadernillo utilizando un lapicero. Se utilizan algoritmos y técnicas de procesamiento de imágenes como el filtrado espacial, umbralización, segmentación, descripción y reconocimiento a través de redes neuronales. El método propuesto permite automatizar el ingreso de notas académicas a una base de datos, sin alterar significativamente el formato de los cuadernillos utilizado en las evaluaciones de los estudiantes. Los resultados obtenidos demuestran que el método propuesto alcanza un error máximo del $2 \%$ en el proceso de reconocimiento.
\end{abstract}

Palabras clave: procesamiento digital de imágenes, redes neuronales 


\section{Introducción}

En una institución académica que alberga gran cantidad de alumnado, el registro de notas de las distintas evaluaciones de las asignaturas que se desarrollan durante el ciclo académico resulta complejo y demanda mucho tiempo. A diferencia de otros sistemas que utilizan lectores ópticos para la lectura de las "marcas" (Sistema feedbacK y Lector óptico SR 11000) el método propuesto utiliza una cámara web.

En la literatura científica se han encontrado trabajos anteriores que apuntan a resolver problemas similares al planteado en el presente documento. En la mayoría de los casos están enfocados al reconocimiento directo de números en lugar de marcas ópticas codificadas.

Por ejemplo, Batuwita y Bandara (2006) proponen el reconocimiento de números escritos a mano en imágenes digitales utilizando lógica difusa, debido a que con un sistema difuso obtienen flexibilidad, eficiencia y capacidad de adaptación. Las imágenes son adquiridas con un escáner de papel. El porcentaje de acierto de este método varía, para algunos números el acierto va hasta el $50 \%$ y para otros hasta el 100\%. Justamente esta tasa de acierto variable es la desventaja de dicho método.

Kimura y Shridhar (1991) comentan sobre el reconocimiento de números escritos a mano mediante la mezcla de dos algoritmos. Uno de los algoritmos emplea una función discriminante cuadrática modificada, en la que se utiliza la dirección de las características espaciales del número, y el segundo utiliza las características derivativas del perfil del número, logrando tasas de rechazo por debajo del $4 \%$. Esta tasa va a depender mucho del hardware utilizado para la captura de los datos y de la implementación adecuada de los algoritmos propuestos.

El método propuesto se adapta a las necesidades de una institución académica estableciendo el reconocimiento de marcas como esquema de identificación de números. En las siguientes secciones se describen los detalles del método propuesto. Al final se presentan los resultados correspondientes debidamente comentados.

\section{Descripción del método propuesto}

En la Figura 1 se muestra el diagrama de bloques del método propuesto. Primero, a través de una cámara web se adquieren las áreas de interés donde se encuentran las marcas ópticas que indican el código y la nota asignada al alumno. Luego, a través de técnicas de procesamiento digital de imágenes (filtrado, umbralización y segmentación) se obtienen patrones que describen las "marcas" correspondientes. Finalmente, estos patrones se ingresan a una red neuronal que reconocerá el código y la nota del alumno.

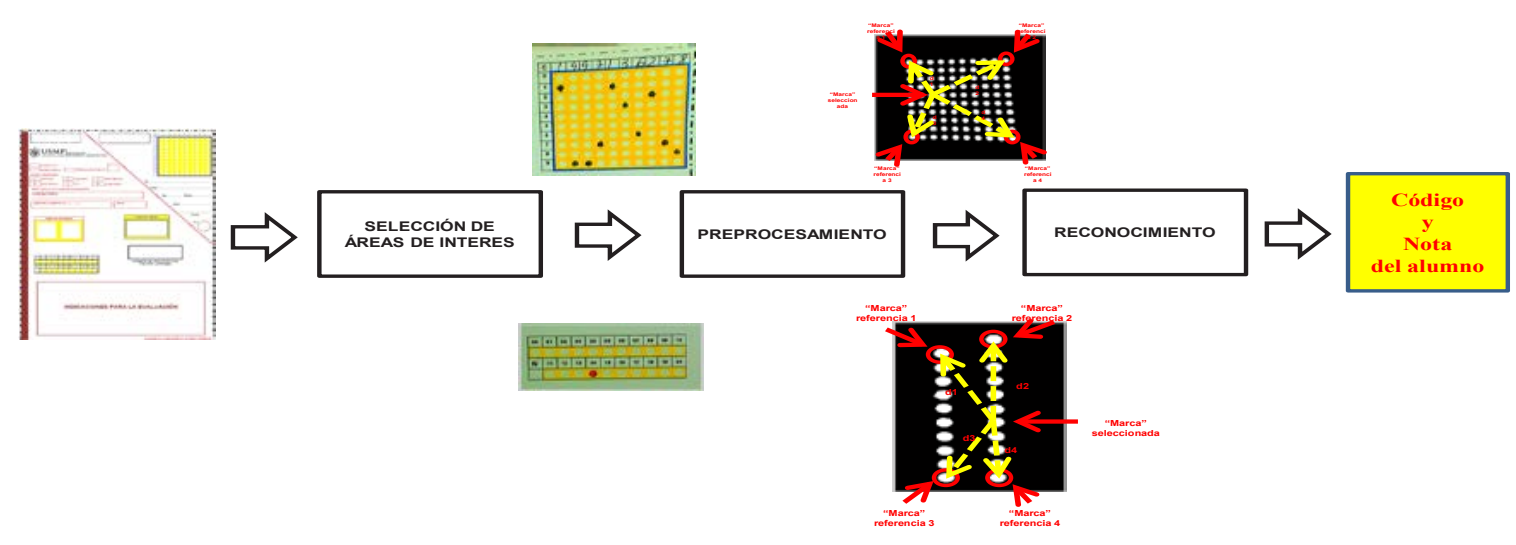

Figura 1. Diagrama de bloques del método propuesto 
Las marcas ópticas deben ser generadas correctamente, es decir, se debe sombrear toda la marca dentro del área correspondiente. En este caso pueden ser llenadas con tinta liquida o seca de color rojo, azul o negro. En el caso de la cuadrícula para el llenado del código, solo debe existir una marca por columna. En el caso de la cuadrícula para el llenado de la nota, solo deberá existir una única marca.

Uno de los objetivos que se planteó en el presente trabajo fue el de desarrollar una nueva carátula del cuadernillo de evaluación que se ajuste a los requerimientos particulares necesarios para la detección. Se procuró que esta propuesta tenga los menores cambios posibles con respecto al cuadernillo utilizado (se mantienen los mismos campos y el mismo tamaño). Por este motivo se tuvo bastante interacción con el coordinador académico de la Facultad de Ingeniería y Arquitectura de la Universidad de San Martín de Porres para que evaluara las diferentes propuestas. En la Figura 2 se muestra el cuadernillo actual que se utiliza para las distintas evaluaciones.

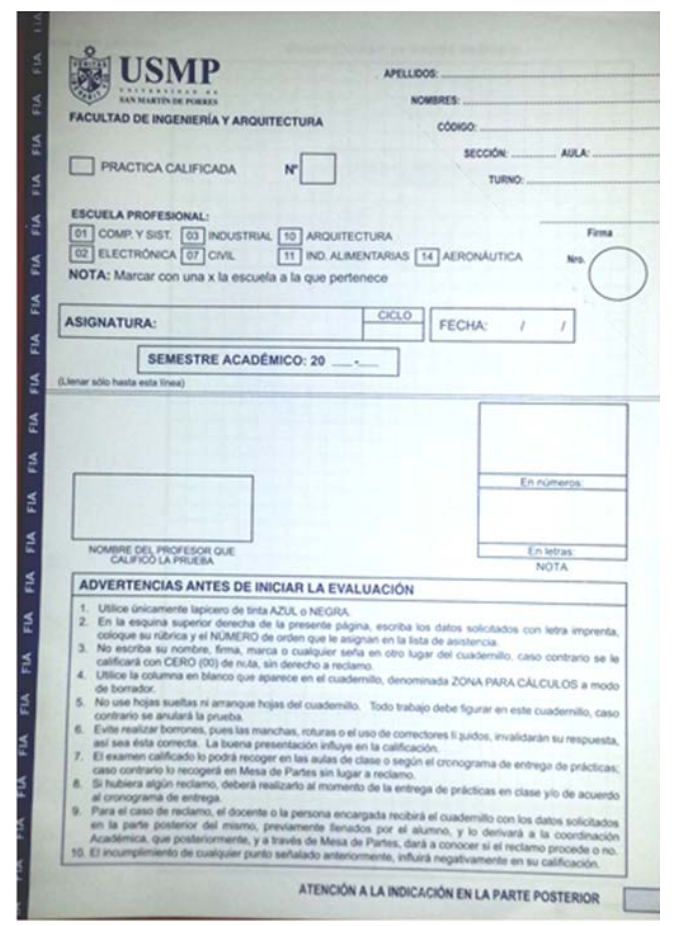

Figura 2. Cuadernillo de evaluación actual
En la Figura 3 se muestra la carátula del cuadernillo de evaluación propuesto. $\mathrm{Al}$ iniciar el proceso se seleccionan dos áreas de procesamiento o de interés. De esa manera, se concentra el procesamiento en las áreas específicas. Dichas áreas son: "nota del alumno en marcas" y "código del alumno en marcas". Está selección solo se realiza una vez al inicio del proceso y se guardan las posiciones de dichas áreas. El cuadernillo de evaluación donde se encuentran las áreas de interés se muestran en la Figura 4.

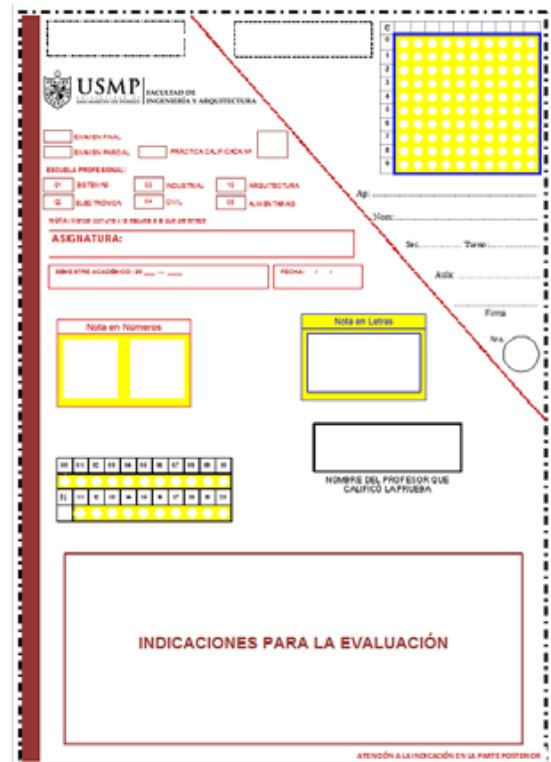

Figura 3. Cuadernillo de evaluación diseñado

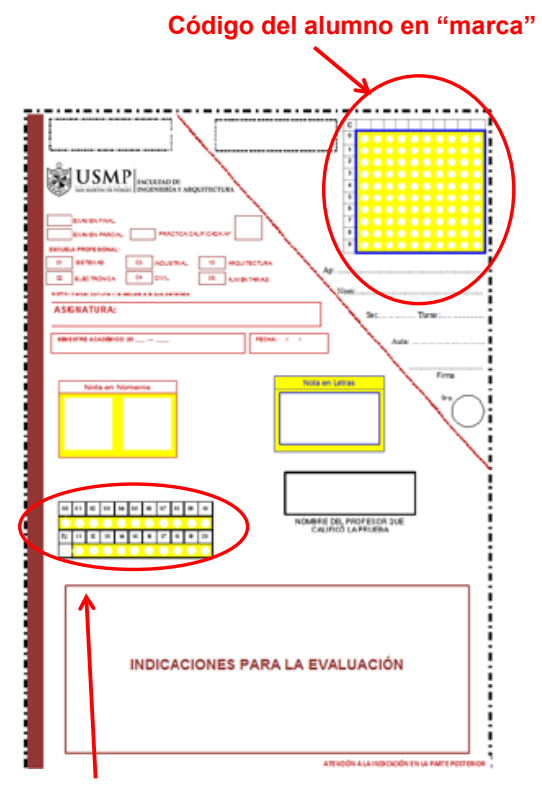

Nota del alumno en "marca"

Figura 4. Áreas de interés del cuadernillo a procesar 
En la Figura 5, se muestra la cuadrícula donde el alumno coloca su código. En la fila superior, el alumno coloca en números su código (que está compuesto de 10 dígitos) y sombrea cada marca blanca que corresponde al dígito del código. En la Figura 6 se muestra un ejemplo de ello:

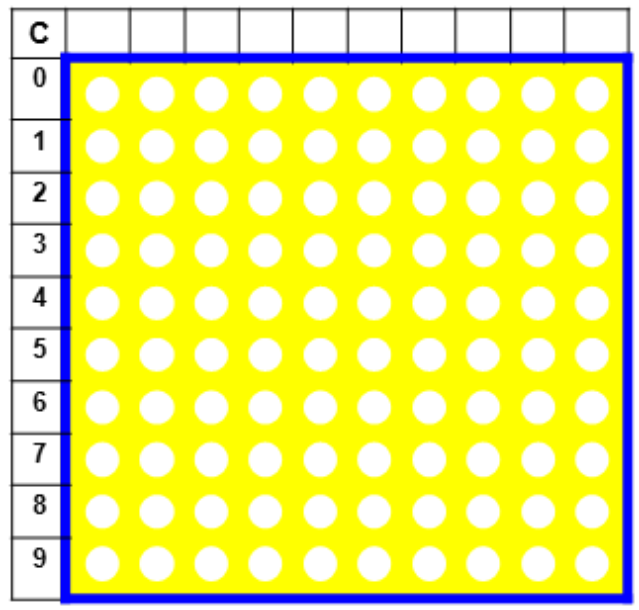

Figura 5. Cuadrícula para colocar el código del alumno en marcas

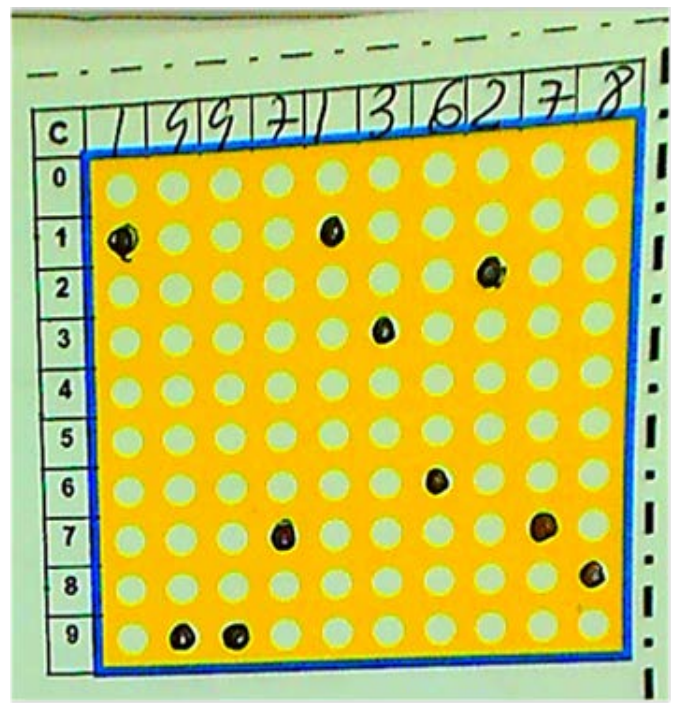

Figura 6. Ejemplo del código del alumno

En la Figura 7, se muestra la cuadrícula donde el docente que califica el examen coloca la nota correspondiente en "marca". En este caso, solo se sombrea la marca blanca que corresponde a la nota asignada. Cabe resaltar que la nota será un número entero. En la Figura 8, se muestra un ejemplo de ello.

\begin{tabular}{|l|l|l|l|l|l|l|l|l|l|l|}
\hline 00 & 01 & 02 & 03 & 04 & 05 & 06 & 07 & 08 & 09 & 10 \\
\hline \multicolumn{10}{|c|}{} \\
\hline N & 11 & 12 & 13 & 14 & 15 & 16 & 17 & 18 & 19 & 20 \\
\hline
\end{tabular}

Figura 7. Cuadrícula para colocar la nota del alumno en marcas

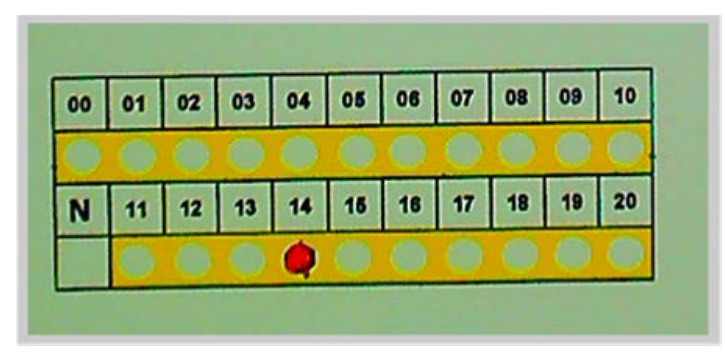

Figura 8. Ejemplo de la nota del alumno

\section{A. Preprocesamiento del código del alumno en marcas}

A continuación, se describe el pre-procesamiento que se realiza a las áreas de interés, "código del alumno en marcas" y "nota del alumno en marcas".

La imagen del área de interés "código del alumno en marcas" se denotada como " $I_{1}$ ".

La etapa de pre-procesamiento de la imagen del área de interés "código del alumno en marcas" se inicia con el proceso de umbralización de las componentes primarias de color:

$I_{1 B D}(x, y)=\left\{\begin{array}{cc}255, & I_{1 R}(x, y) \geq \mu_{0} \wedge \\ & I_{1 G}(x, y) \geq \mu_{1} \wedge \\ & I_{1 B}(x, y) \geq \mu_{2} \\ 0, & \text { caso contrario }\end{array}\right.$

Donde " $I_{1 R}$ ” " $I_{1 G}$ ” $\mathrm{e}$ “ $I_{1 B}$ ” son respectivamente las componentes primarias Rojo, Verde y Azul de la imagen " $I_{1}$ ”.

" $I_{I B D}$ " constituye la imagen resultante de la umbralización. 
Es importante resaltar que el color amarillo facilita el proceso de segmentación y extracción del área de interés. En ese sentido a partir de las distintas pruebas realizadas, se encontraron niveles de umbralización apropiados: $\mu_{0}=75, \mu_{1}=65$ y $\mu_{2}=0$.

El resultado de la umbralización se muestra en la Figura 9.
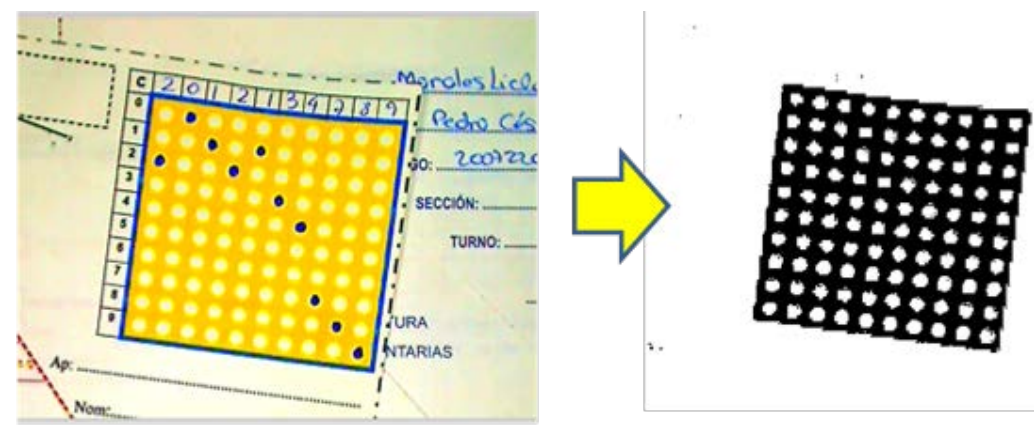

Figura 9. Imagen en formato RGB del área "código del alumno en marca" y su imagen binarizada resultante

A continuación, a la imagen " $I_{1 B D}$ " se le aplicará el algoritmo de etiquetado. Hay que tener en cuenta que dicho algoritmo tomará como etiqueta a los pixeles que son de color blanco, por lo tanto, en dicha imagen existirán 100 etiquetas (las 100 marcas). La imagen que contiene las etiquetas será “ $I_{1 B E}$ ” y se muestra en la Figura 10.
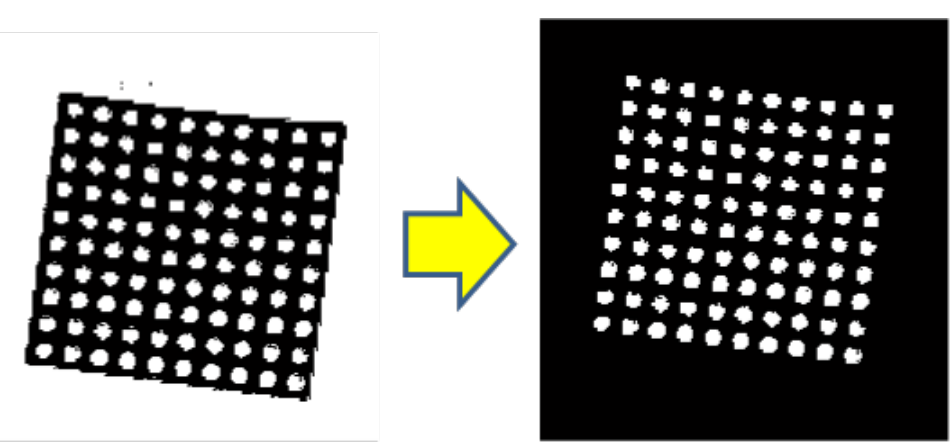

Figura 10. Imagen resultante del proceso de etiquetado

Como se puede apreciar en la Figura 10 aparecen todas las "marcas" indistintamente de que si se han sombreado o no.

Las "marcas" sombreadas, se obtendrán de la imagen " $I_{1 R}$ ” (componente $\mathrm{R}$ de $I_{1}$ ). En esta imagen es fácil segmentar las no sombreadas (Figura 11). Para este proceso se aplica la siguiente expresión considerando $\mu_{3}=171$ :

$$
I_{1 R B}(x, y)= \begin{cases}0, & I_{1 R}(x, y) \geq \mu_{3} \\ & I_{1 B E}(x, y)>0 \\ & \\ 255, & \text { otra forma }\end{cases}
$$

Como se puede observar la umbralización se aplica solo a las posiciones de las etiquetas de la imagen " $I_{1 \mathbb{E}}$ ”. El resultado que se obtiene será una imagen binaria que no contenga las marcas sombreadas. Esto se aprecia en la Figura 12. 

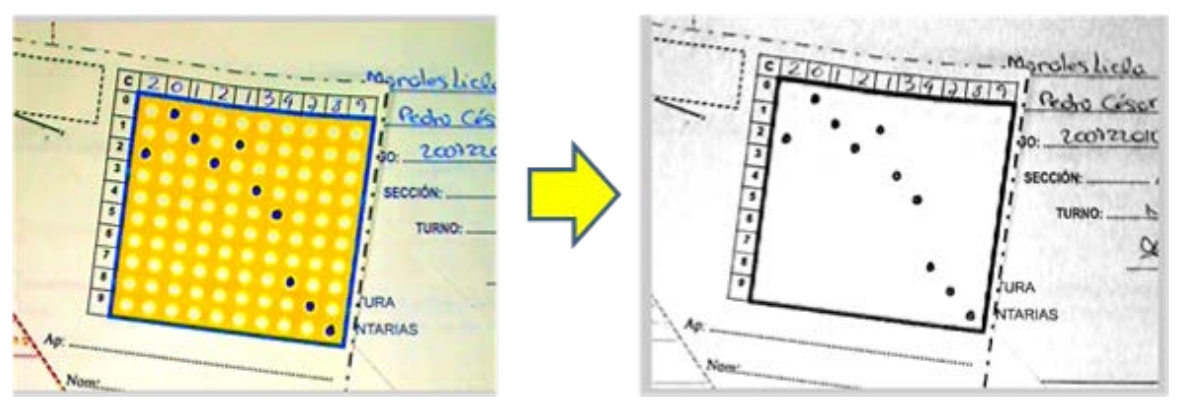

Figura 11. Componente "R" de la imagen del área de código del alumno en marcas
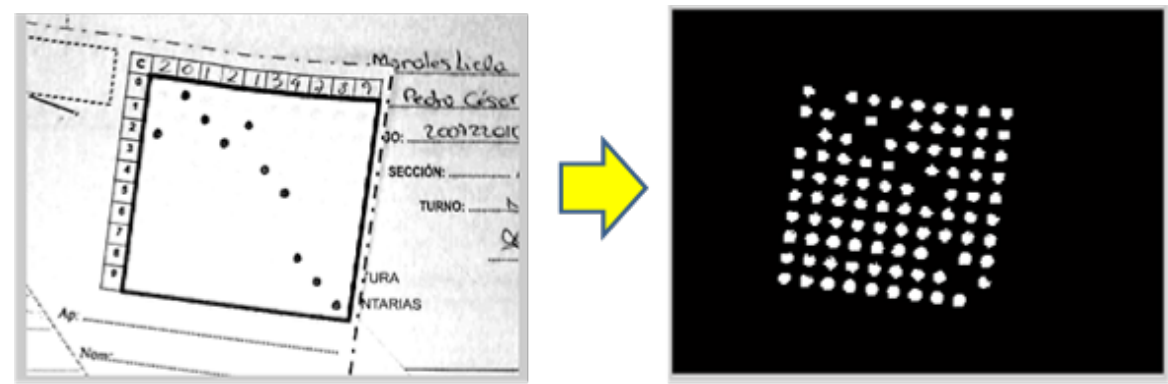

Figura 12. Imagen $I_{1 R B}$ resultante

Una vez obtenido la imagen " $I_{1 \mathbb{B}}$ ” $\mathrm{e}$ “ $I_{1 R B}$ " se aplica la operación lógica OR- EXCLUSIVA dando como resultado la imagen " $I_{1 B C}$ ".
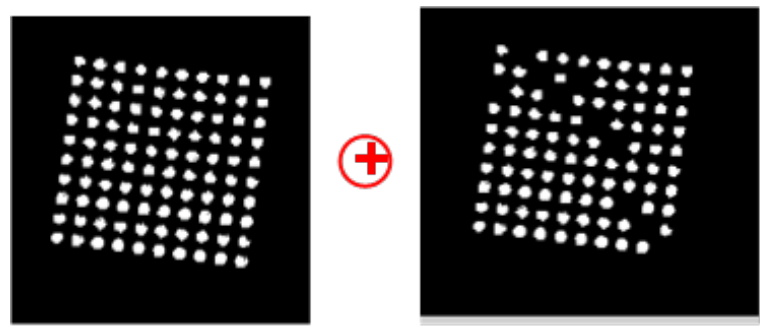

$$
I_{1 B C}=I_{1 B D} \oplus I_{1 R B}
$$

La imagen resultante se aprecia en la Figura 13 .

Figura 13. Proceso de obtención de las "Marcas" seleccionadas

Denominamos “ $I_{1 B C_{k}}$ ” como la imagen (obtenida de " $I_{1 B C}$ ”) que contiene únicamente al objeto con etiqueta "k". Estas etiquetas corresponderán a cada una de las "marcas" que forman el código del alumno. El centroide (Cheriet, Kharma, Liu \& Suen, 2007) de cada una de estas etiquetas se obtiene a partir del momento de orden cero y los momentos de orden uno y de " $I_{1 B C_{k}}$ ":

$$
\begin{gathered}
M_{k}(0,0)=\sum_{x=0}^{N-1} \sum_{y=0}^{M-1} I_{1 B C_{k}}(x, y) \\
M_{k}(1,0)=\sum_{x=0}^{N-1} \sum_{y=0}^{M-1} x \cdot I_{1 B C_{k}}(x, y) \\
M_{k}(0,1)=\sum_{x=0}^{N-1} \sum_{y=0}^{M-1} y \cdot I_{1 B C_{k}}(x, y)
\end{gathered}
$$

Luego el centro de masa se obtiene a partir de las siguientes expresiones: 


$$
\begin{aligned}
& x_{k}=M_{k}(1,0) / M_{k}(0,0) \\
& y_{k}=M_{k}(0,1) / M_{k}(0,0)
\end{aligned}
$$

donde " $x_{k}$ " e " $y_{k}$ " son las coordenadas del centro de masa de cada marca seleccionada.

El descriptor (6) que se utilizará para reconocer cada "marca seleccionada" será la distancia euclidiana que existe entre las "marcas seleccionadas" y los cuatro extremos que conforma el grupo de "marcas" de referencia “ $x_{r}$ ” e " $y_{r}$ " (Figura 14).

La matriz de distancias euclidianas es obtenida a partir de la siguiente expresión:

$$
D C 1_{r k}=\sqrt{\left(x_{k}-x_{r}\right)^{2}+\left(y_{k}-y_{r}\right)^{2}}
$$

donde $k=1,2,3, \ldots, 10$ y $r=1,2,3,4$.

Esto quiere decir que cada "marca" tendrá cuatro (04) distancias que la caracterizan. De esa forma se llamará DC1 a la matriz que contenga las distancias del código del alumno.

La matriz DC1 es una matriz de $4 \times 10$, debido a que son 10 marcas sombreadas y cada una con sus cuatro distancias correspondientes. Este será el descriptor que posteriormente permitirá el reconocimiento del código del alumno.

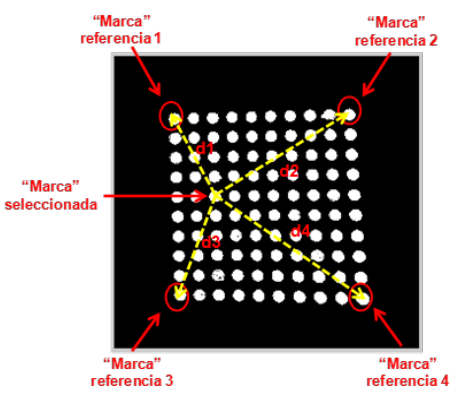

Figura 14. Distancias de cada "marca"

\section{B. Preprocesamiento de la nota del alum- no en marcas}

La imagen del área de interés "nota del alumno en marcas", es segmentada como imagen " $I_{2}$ ”.

La etapa de pre-procesamiento de la imagen se inicia aplicando la ecuación la siguiente umbralización:

$$
I_{2 B D}(x, y)= \begin{cases}255, & I_{2 R}(x, y) \geq \mu_{0} \wedge \\ & I_{2 G}(x, y) \geq \mu_{1} \wedge \\ & I_{2 B}(x, y) \geq \mu_{2} \\ 0, & \text { caso contrario }\end{cases}
$$

Donde " $I_{2 B D}$ " es la imagen binarizada resultante, mientras que “ $I_{2 R}$ ”, “ $I_{2 G}$ ” e " $I_{2 B}$ ” las imágenes correspondientes a las componentes primarias rojo, verde y azul de la imagen " $I_{2}$ ”.

El resultado de la conversión se muestra en la Figura 15.
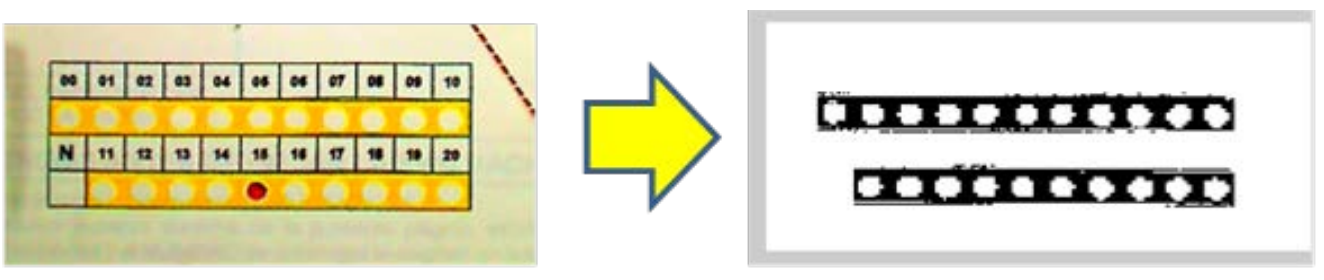

Figura 15. Imagen en formato RGB del área "nota del alumno en marca" y su imagen binarizada resultante

A la imagen " $I_{2 B D}$ " se aplica el proceso de dilatación para poder darle a la imagen mayor consistencia. La imagen resultante es " $I_{2 B D D}$ " y es mostrada en la Figura 16. 


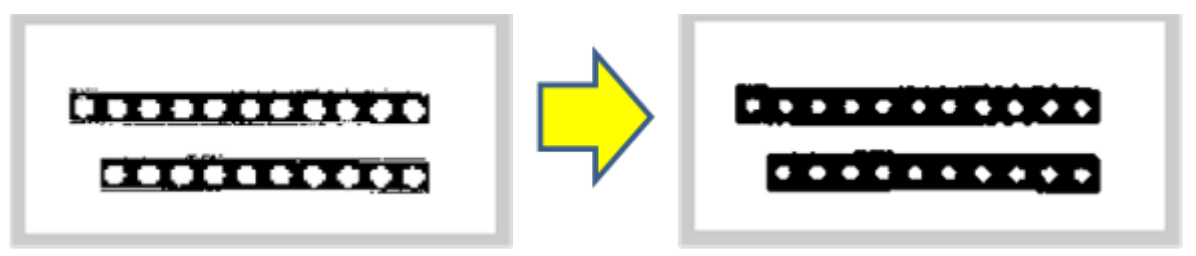

Figura 16. Imagen resultante del proceso de dilatación

A continuación, a la imagen " $I_{2 B D D}$ " se le aplicará el algoritmo de etiquetado. Hay que tener en cuenta que dicho proceso tomará como etiqueta a los pixeles que son de color blanco, por lo tanto, en dicha imagen existirán 21 etiquetas (las marcas). La imagen que contiene las etiquetas en color blanco será “ $I_{2 B D E}$ " y es mostrada en la Figura 17.

\section{$\cdots \cdots \cdots \cdots+\bullet$ \\ $\cdots \cdots \cdots+\cdots$}

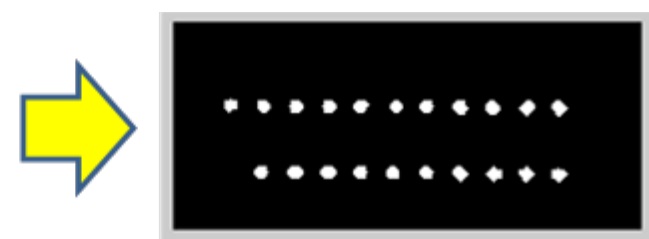

Figura 17. Imagen resultante

Como se puede apreciar en la figura 17 aparecen todas las "marcas" indistintamente de que si se han sombreado o no.
Las “marcas” sombreadas se obtendrán de la imagen " $I_{2 R}$ ", en donde sobresalen de las no sombreadas (Figura 18). Para este proceso se aplica (12), siendo el umbral $\mu_{4}=171$ :

$$
I_{2 R B}(x, y)= \begin{cases}0, & I_{2 R}(x, y) \geq \mu_{4} \wedge I_{2 B D E}(x, y)>0 \\ 255, & I_{2 R}(x, y)<\mu_{4} \wedge I_{2 B D E}(x, y)>0\end{cases}
$$
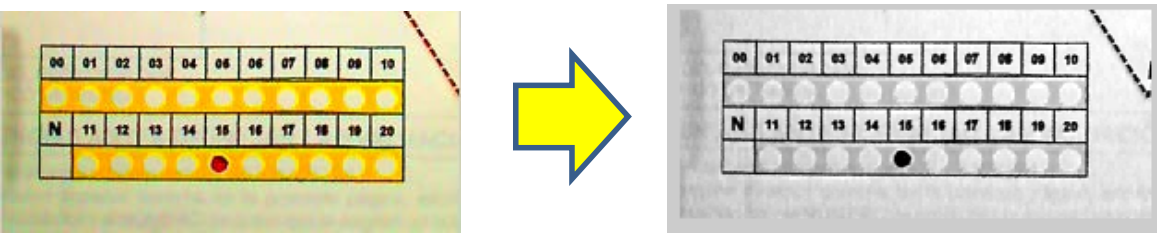

Figura 18. Componente "R" de la imagen del área nota del alumno en marcas

Como se puede observar en la ecuación, este proceso se aplica solo sobre las posiciones de las etiquetas de la imagen " $I_{2 B D E}$ ".
El resultado que se obtiene será una imagen binaria que no contenga las marcas sombreadas. Esto se aprecia en la Figura 19.
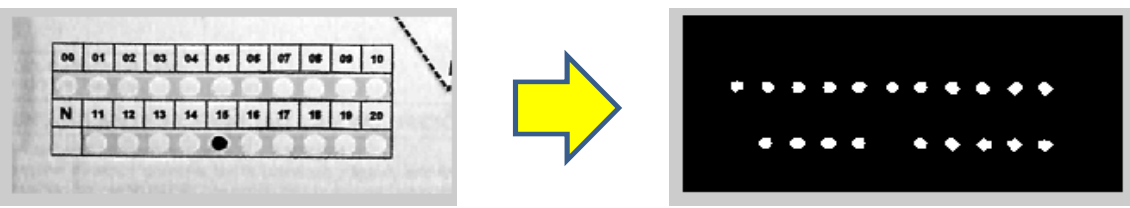

Figura 19. Imagen MS2 resultante 
Una vez obtenido la imagen " $I_{2 \mathbb{B}}$ " e " $I_{2 R B}$ " se aplica la operación lógica OR EXCLUSIVA dando como resultado la imagen " $I_{2 B N}$ ”. La

$$
I_{2 B N}=I_{2 B D} \oplus I_{2 R B}
$$
imagen resultante se aprecia en la Figura 20.
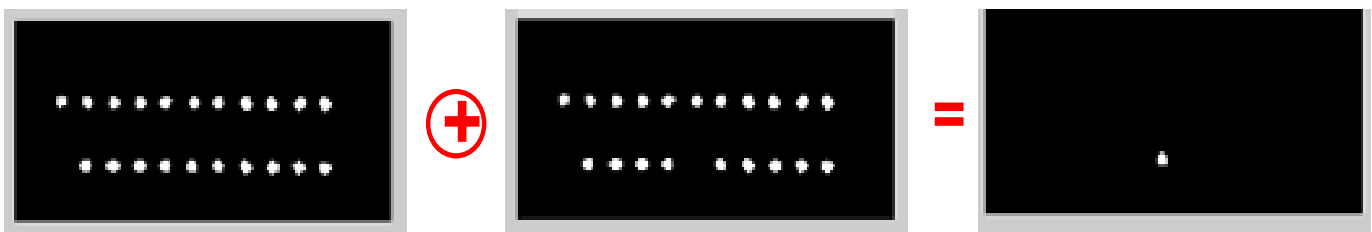

Figura 20. Proceso de obtención de las "Marcas" seleccionadas

Definimos " $I_{2 B N_{1}}$ ” como la imagen que contiene únicamente la etiqueta de la "marca" correspondiente a la nota seleccionada. La posición (centroide) de esta imagen se obtiene a partir de (13), (14, (15), (16) y (17).

$$
\begin{gathered}
M_{1}(0,0)=\sum_{x=0}^{N-1} \sum_{y=0}^{M-1} I_{2 B N_{1}}(x, y) \\
M_{1}(1,0)=\sum_{x=0}^{N-1} \sum_{y=0}^{M-1} x \cdot I_{2 B N_{1}}(x, y) \\
M_{1}(0,1)=\sum_{x=0}^{N-1} \sum_{y=0}^{M-1} y \cdot I_{2 B N_{1}}(x, y) \\
x_{1}=M_{1}(1,0) / M_{1}(0,0) \\
y_{1}=M_{1}(0,1) / M_{1}(0,0)
\end{gathered}
$$

El descriptor que se utilizará para reconocer cada "marca seleccionada" será la distancia euclidiana (ecuación 18) que existe entre esta "marca" seleccionada con los cuatro extremos que conforma el grupo de "marcas" de referencia como se puede apreciar en la Figura 21 .

$$
\begin{aligned}
D N 1_{r 1}= & \sqrt{\left(x_{1}-x_{r}\right)^{2}+\left(y_{1}-y_{r}\right)^{2}} \\
& \text { donde } r=1,2,3,4 .
\end{aligned}
$$

Donde " $x_{1}$ " $e$ " $y_{1}$ " constituyen el centro de gravedad de cada marca seleccionada $y$ " $x_{r}$ " e " $y_{r}$ " el centro de gravedad de cada marca de referencia.
Esto quiere decir que la "marca" tendrá cuatro (04) distancias que lo caracterizan. Se llamará $\mathrm{DN} 1$ a la matriz que contenga las distancias de la nota del alumno en marcas.

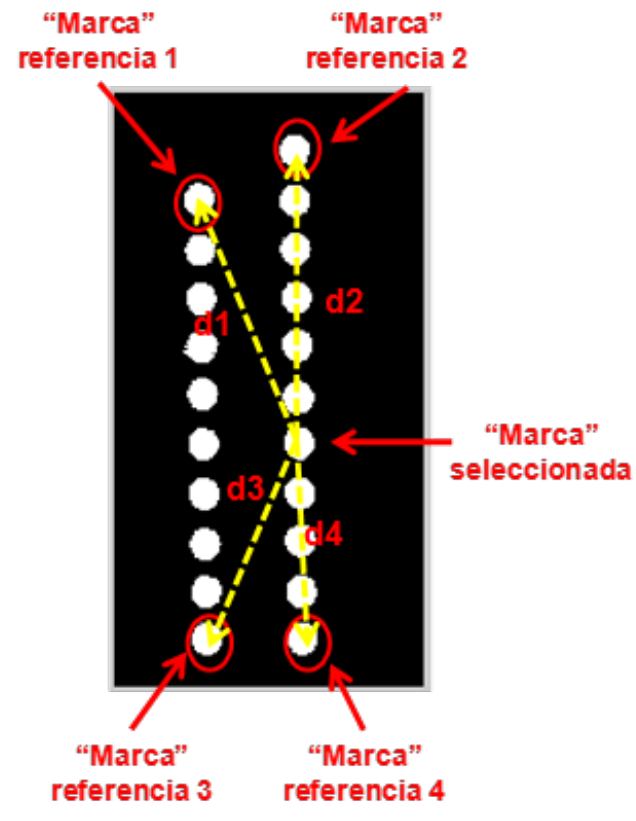

Figura 21. Distancias de cada "marca"

La matriz DN1 es una matriz de $4 \times 1$, debido a que solo es una marca la que se ha sombreado y esta con sus cuatro distancias correspondientes; y este será el descriptor que permitirá el reconocimiento de la nota del alumno en "marcas".

\section{Etapa de reconocimiento}

Para la etapa de reconocimiento del código y la nota del alumno en marcas se hace uso de redes neuronales (Demuth, Beale $\&$ Hagan, 2009). La implementación de la red 
neuronal se utilizó el toolbox de redes neuronales de MATLAB. Para la elección del número de capas ocultas de cada red se utilizó la regla de la pirámide geométrica que se basa en la suposición de que el número de neuronas de la capa oculta ha de ser inferior al total de las variables de entrada, pero superior al número de variables de salida (Masters, 1993). Se considera que el número de neuronas en cada capa sigue una progresión geométrica, tal que, para una red de una única capa oculta, el número de neuronas intermedias debe ser próximo a $\sqrt{N 1 \cdot M 1}$, siendo $\mathrm{N}$ el número de variables de entrada y $\boldsymbol{M} 1$ el total de neuronas de salida.

Si la red tuviera dos capas ocultas, el número de neuronas de la primera capa oculta debería ser próximo a $H_{1}=M 1 \cdot p^{2}$, y el número de neuronas de la segunda capa oculta debería ser próximo a $H_{2}=M 1 \cdot p$ siendo $p=\sqrt[3]{N 1 / M 1}$.

Se usó el algoritmo de entrenamiento de gradiente conjugado escalado "Scaled conjugate gradient", cuya función de entrenamiento es llamada "Trainscg" (Haykin, 1998). Un algoritmo que no requiere bús- queda lineal. Este algoritmo se utilizó para las dos redes que se implementó.

La red neuronal que se utilizó para la detección de la "marca" dentro de la cuadricula código del alumno en marcas, se llamará RN1 y esta tiene una capa de entrada con 4 entradas, primera capa oculta con 30 neuronas, segunda capa oculta con 25 neuronas y una capa de salida con 20 salidas. En ambas capas ocultas se utilizó la función de transferencia sigmoidal.

Las cuatro entradas serán las distancias con que se caracterizaron a cada marca y las 20 salidas indicarán en qué fila (los primeros 10 valores) y columna (los 10 valores restantes) se encuentra la marca detectada.

En la Figura 22 se muestra un ejemplo. Como se aprecia para los siguientes valores de entrada se obtiene que la marca detectada se encuentre en la fila 6 y columna 3 . El código de alumno está compuesto por 10 dígitos, por lo tanto, la cuadrícula del código del alumno que contiene 100 marcas, tendrá 10 de estas sombreadas como muestra la Figura 6.

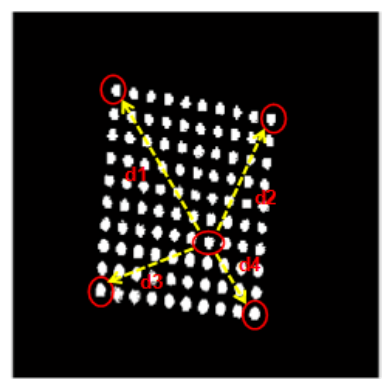

a)

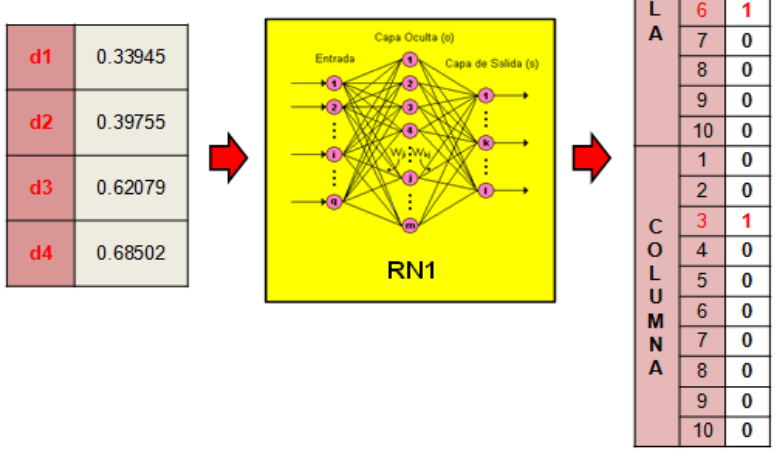

b)

Figura 22. Ejemplo de reconocimiento de RN1 
Entonces, para el reconocimiento se necesita la matriz donde se encuentre las distancias de estas 10 marcas. Estas, se obtuvieron en la etapa de pre procesamiento y se guardaron en la matriz $D C 1$.

A la red neuronal que se utilizó para la detección de la "marca" dentro de la cuadrícula "nota del alumno en marcas", se le denominará RN2. Esta tiene una capa de entrada con 4 entradas, la primera capa oculta con 35 neuronas, la segunda capa oculta con 25 neuronas y la capa de salida con 21 salidas. Las cuatro entradas serán las distancias con que se caracterizó a la marca y las 21 salidas indicarán la nota que fue seleccionada. En ambas capas ocultas se utilizó la función de transferencia sigmoidal.

En la Figura 23, se muestra un ejemplo. Como se aprecia para los siguientes valores de entrada se obtiene que la marca detectada sea 12 .

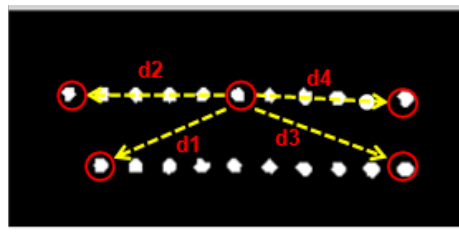

a)
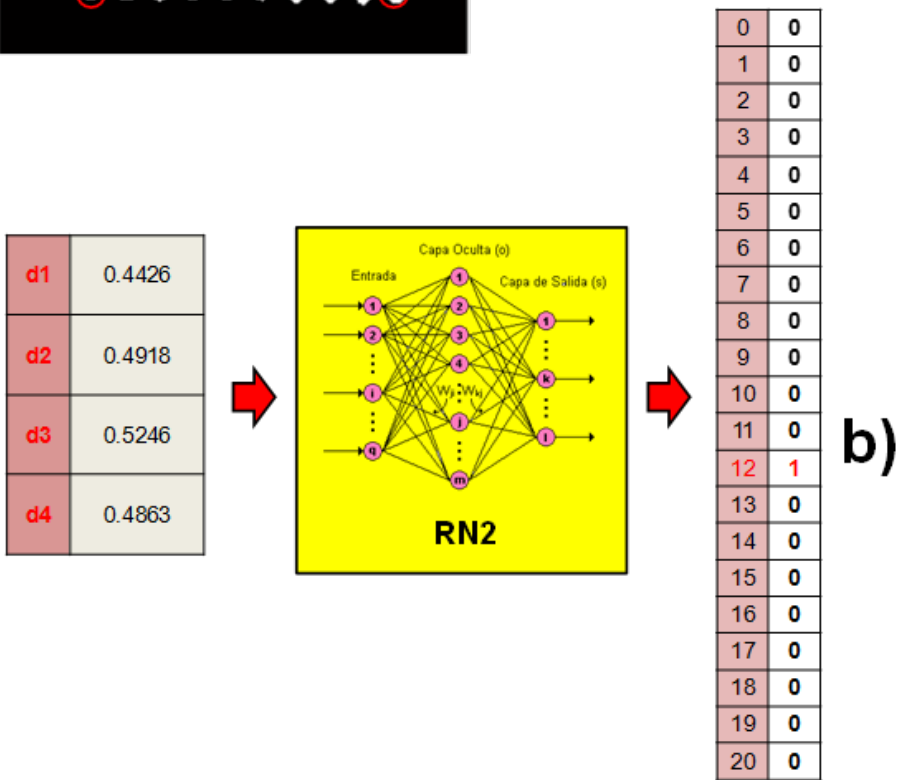

Figura 23. Ejemplo de reconocimiento RN2

La nota del alumno está compuesta por un solo valor, por lo tanto, la cuadrícula de nota del alumno que contiene 21 marcas, tendrá solo una de estas sombreada como muestra la Figura 8. Entonces para el reconocimiento se necesita la matriz donde se encuentren las distancias de esta marca. Estas se obtuvieron en la etapa de pre procesamiento y se guardaron en la matriz DN1.

\section{Resultados}

Las pruebas se han realizado sobre un recinto mostrado en la Figura 24. Dicho recinto cuenta con una iluminación provista de seis indicadores LEDs de alta intensidad de 3.5 vatios cada uno. La disposición de estos LEDs es mostrada en la Figura 25. El esquema permite que se tenga una iluminación constante y homogénea sobre el cuadernillo. 
El recinto también cuenta con una cámara para la adquisición de las imágenes de los cuadernillos a procesar (que son jalados secuencialmente desde una bandeja de carga y posicionados debajo de la cámara por un sistema electromecánico). En cada adquisi- ción, el sistema permite mantener una distancia constante de $25 \mathrm{~cm}$ entre la cámara y el cuadernillo. Esto permite que todas las tomas sean adquiridas con una resolución espacial uniforme.
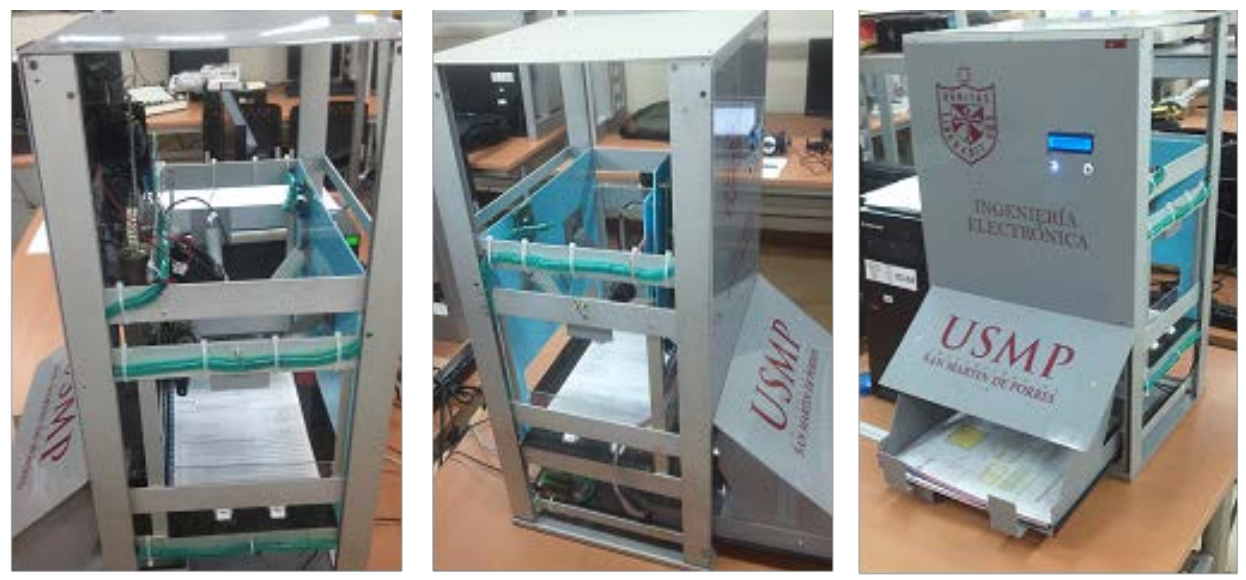

Figura 24. Fotos del recinto de adquisición.

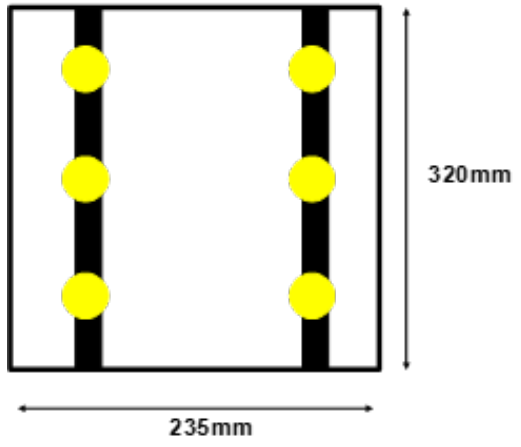

Figura 25. Diagrama de arreglo de LEDs

Para la correcta detección se tiene que cumplir con lo siguiente: el cuadernillo debe estar posicionado correctamente; el recinto debe estar iluminado; finalmente las marcas deben estar debidamente sombreadas, es decir, no muy tenues y dentro del espacio correspondiente.

Para la validación del código y nota del alumno en marcas, se utilizaron las ecuaciones (19) y (20) para determinar los verdaderos reconocimientos de códigos y notas. Fueron 50 cuadernillos (debidamente sombreados en las marcas) que se procesaron cinco veces.

$$
\begin{gathered}
P_{a c}=\frac{\text { Cantidad de codigos reconocidos }}{\text { Cantidad total de codigos } \times \text { Cantidad de veces procesados }} \times 100 \\
P_{a n}=\frac{\text { Cantidad de notas reconocidos }}{\text { Cantidad total de notas } \times \text { Cantidad de veces procesados }} \times 100
\end{gathered}
$$

En el caso de las marcas, para el código y nota, el error que se presentó fue debido a que no se sombreó correctamente la marca o se sombreó fuera de la marca.
En laTabla 1, se muestra los resultados obtenidos del reconocimiento de las marcas, tanto del código del alumno como de la nota, por medio de redes neuronales. 
Tabla 1

Resultados de reconocimiento de los códigos y notas mediante marcas haciendo uso de redes neuronales

\begin{tabular}{|c|c|c|c|c|c|}
\hline & $\begin{array}{c}\text { VERDADERO } \\
\text { RECONOCIMIENTO }\end{array}$ & $\begin{array}{c}\text { FALSO } \\
\text { RECHAZO }\end{array}$ & UNIVERSO & $\begin{array}{c}\mathbf{N}^{\circ} \text { DE } \\
\text { VECES }\end{array}$ & $\begin{array}{c}\mathbf{N}^{\circ} \text { total de } \\
\text { pruebas }\end{array}$ \\
\hline CÓDIGO & $98.4 \%$ & $1.6 \%$ & 50 & 5 & 250 \\
\hline NOTA & $98.8 \%$ & $1.2 \%$ & 50 & 5 & 250 \\
\hline
\end{tabular}

\section{Conclusiones}

Se diseñó una nueva carátula del cuadernillo de evaluación que cumplió con los requerimientos del sistema.

Al realizar el procesamiento de marcas ópticas mediante una cámara web, se suple el uso de un lector óptico de marcas. Esto permite un ahorro considerable en un sistema de registro automático de evaluaciones debido al costo de dichos lectoras.

\section{Referencias}

Batuwita, K., \& Bandara, G. (June, 2006). Fuzzy Recognition of Offline Handwritten Numeric Characters. IEEE Conference on Cybernetics and Intelligent Systems, Bangkok, 1-5, doi: 10.1109/ICCIS.2006.252356.

Cheriet, M., Kharma, N., Liu, C., \& Suen, C. (2007). Character Recognition Systems - A guide for Students and Practioners. New Jersey: John Wiley \& Sons.

Demuth, H., Beale, M. \& Hagan, M. (2009). Neural Network Toolbox ${ }^{\mathrm{TM}} 6$ User's Guide. Recuperado de http:// filer.case.edu/pjt9/b378s10/nnet.pdf

Haykin, S. (1998). Neural Networks: A Comprehensive Foundation ( $2^{\text {nd }}$ Ed.). USA: Prentice Hall.
Kimura, F., \& Shridhar, M. (1991). Handwritten numerical recognition based on multiple algorithms. Pattern Recognition Society, 24(10), 969-983.

Masters, T. (1993). Practical Neural Network Recipes in C++. San Diego, CA: Academic Press.

Sistema feedback [Equipos y software]. (2015). Argentina: feedback.

SR 11000 [Lector óptico]. Japón: SEKONIC OMR. 
